\title{
Numerical Solution of Non-Linear Prey-Predator System using Finite Elements Method
}

Saad A. Manaa

Ahmed F. Qasem

College of Computer sciences and Mathematics

University of Mosul, Iraq

Received on: 21/02/2007

Accepted on: 16/04/2007

\section{ABSTRACT}

A non-linear prey-predator system solved numerically by Galerkin method, and we compare these results with the results of Pius Peter Nyaanga[6] who used finite difference methods, we found that Galerkin finite elements method is faster than finite difference method to reach equilibrium state where the density for the prey $u(x, t)$ and the predator $v(x, t)$ are equals for all the values for $x$ and $t$, also we found that Galerkin method converges towards the steady state solutions faster than finite difference method with less steps in time.

Keywords: prey-predator system, Galerkin method, finite difference methods.

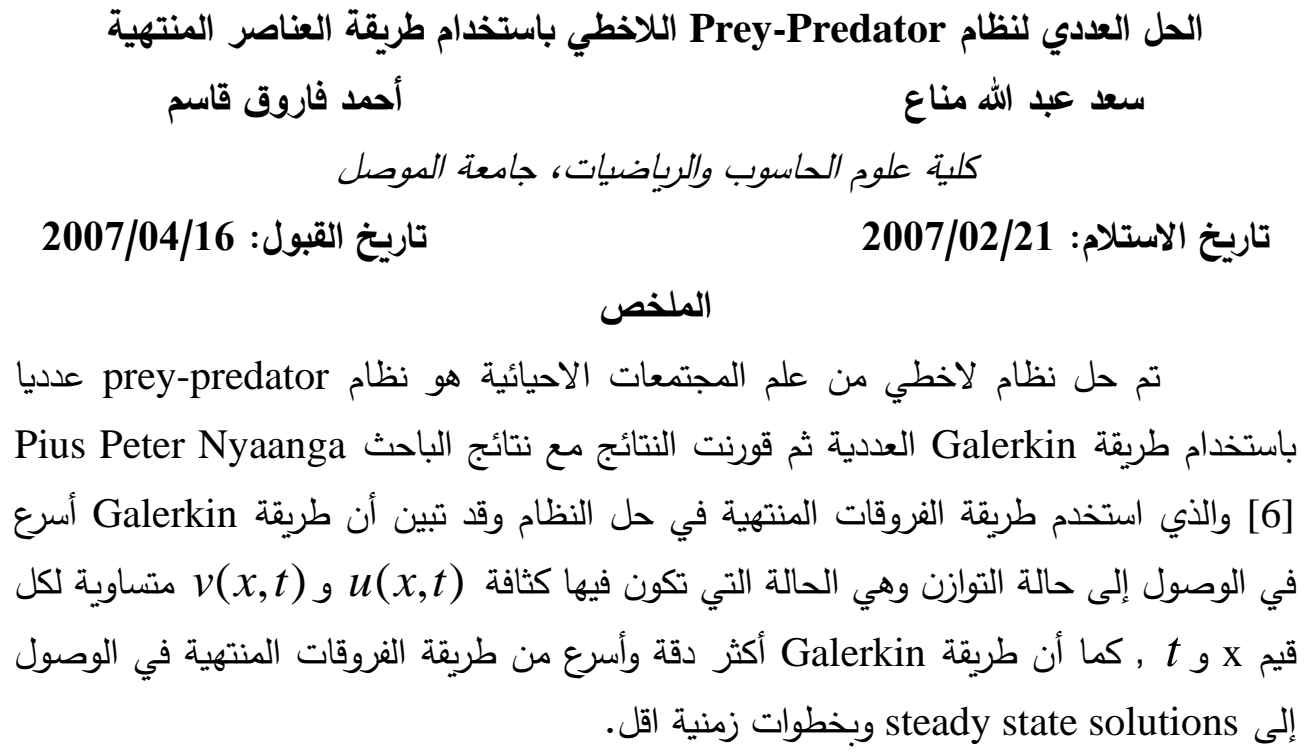

الكلمات المفتاحية: نظام Grey-predator، طريقة Galerkin، طريقة الفروقات المنتهية.

\section{Introduction:}

The finite element method is one of the most flexible tools available for solving engneering problems of the kind involved in analyzing the deformation of solids, the transfer of heat, the flow of fluids, or electrical problems, it can be applied to systems with virtually any geometric configuration or boundary conditions.[1] 
The finite element method has developed simultaneously with the increasing use of high-speed electronic digital computers and with the growing emphasis on numerical methods for engineering analysis. Although the method was originally developed for structural analysis, the general nature of the theory on which it is based has also made possible its successful application for solutions of problems in other fields of engineering[3].

A basic model for studying the interraction of species in population biology is the prey-predator model, the prey-predator model is a planar system representing the behavior of a population of prey, as fish, and a population of predators, as sharks[6].

$\mathrm{Du}$ and Lou [4] studied some uniqueness and exact multiplicity results for a predator-prey model, they consider positive solutions of a predator-prey model with diffusion and under homogeneous dirichlet boundary conditions, it turns out that a certain parameter in this model plays a very important role.

Tyutyunov et.al [2] studied directed movement of predators and the emergence of density-dependence in predator-prey models. Numerical analysis shows that, on the spatially aggregated scale, the average predator density adversely affects the individual consumption, leading to a non-linear predator-dependent trophic function.

Meng and Wang [5] studied asymptotic behavior of a predator-prey diffusion system with time delays. They found that the global asymptotic convergence is established by the upper-lower solutions and iteration method in terms of the rate constants of the reaction function independent of the time delays and the effect of diffusion.

In this paper, we will study the numerical solution for prey-predator system by Galerkin method and we will compare these results with the results of [6].

\section{Mathematical model:}

A basic model for studying the interraction of species in population biology is the prey-predator model, the prey-predator model is [6]:

$$
\begin{aligned}
& \frac{\partial \mathrm{u}}{\partial \mathrm{t}}=\mathrm{D}_{1} \frac{\partial^{2} \mathrm{u}}{\partial \mathrm{x}^{2}}+\alpha \mathrm{u}(1-\mathrm{u})-\mathrm{k}_{1} \mathrm{uv} \\
& \frac{\partial \mathrm{v}}{\partial \mathrm{t}}=\mathrm{D}_{2} \frac{\partial^{2} \mathrm{v}}{\partial \mathrm{x}^{2}}-\mu \mathrm{v}+\mathrm{k}_{2} \mathrm{uv}
\end{aligned}
$$

where $\mathrm{u}=\mathrm{u}(\mathrm{x}, \mathrm{t})$ represents the number of fish (prey), $\mathrm{v}=\mathrm{v}(\mathrm{x}, \mathrm{t})$ is the number of sharks (predator),

$\left(D_{1}\right)$ is diffusion coefficient of the prey, 
$(\alpha)$ is the rate of growth of the prey,

$\left(D_{2}\right)$ is diffusion coefficient of the predator,

$(\mu)$ is positive constant and represents the death rate of the predator,

$k_{1}, k_{2}$ are positive constants and they represent the rate of interraction between the prey and the predator which facilitates the killing of prey by predator.

With initial conditions:

$\left.\begin{array}{ll}\mathrm{u}(\mathrm{x}, 0)=\mathrm{f}(\mathrm{x}) & \mathrm{x} \in[0, \mathrm{~L}] \\ \mathrm{v}(\mathrm{x}, 0)=\mathrm{g}(\mathrm{x}) & \mathrm{x} \in[0, \mathrm{~L}]\end{array}\right\}$

here

$0 \leq \mathrm{f}(\mathrm{x})$

for all $x \in[0, L]$

$0 \leq \mathrm{g}(\mathrm{x})$

for all $x \in[0, L]$

and Neumann boundary conditions:

$\frac{\partial \mathrm{u}}{\partial \mathrm{x}}=0$

at $\mathrm{x}=0$ and $\mathrm{x}=\mathrm{L} \quad$ \}

$\frac{\partial v}{\partial x}=0$

at $\mathrm{x}=0$ and $\mathrm{x}=\mathrm{L}$

\section{Numerical solution:}

Galerkin's method has been discussed by several authors[8]. It is a means of obtaining an approximate solution to a differential equation. It does this by requiring that the error between the approximate solution and the exact solution be orthogonal to the functions used in the approximation.

If we start with a differential equation $\mathrm{Lu}-\mathrm{f}=0$ ( $\mathrm{L}$ is a differential operator) and approximate the solution by $\mathrm{u}^{*}=\sum \mathrm{N}_{\mathrm{i}} \mathrm{u}_{\mathrm{i}}$ then the solution $\mathrm{Lu}^{*}-\mathrm{f}=\varepsilon$ where $\varepsilon$ is a residual or error. Our desire is to make $\varepsilon$ as small as possible. One way of accomplishing this objective is to require the integral $\int_{R} N_{i} \varepsilon d x=0$ for each basis function $N_{i}$. This integral mathematically states that the basic function must be orthogonal to the error over the region $\mathrm{R}$ [7].

Let $u^{*}$ and $v^{*}$ have the normal finite element form:

$\mathrm{u} \approx \mathrm{u}^{*}=\sum_{\mathrm{e}=1}^{\mathrm{E}} \mathrm{u}^{(\mathrm{e})}$

$\mathrm{v} \approx \mathrm{v}^{*}=\sum_{\mathrm{e}=1}^{\mathrm{E}} \mathrm{v}^{(\mathrm{e})}$

where the interpolation function are [1]:

$\mathrm{u}^{(\mathrm{e})}=\mathrm{N}_{\mathrm{i}}^{(\mathrm{e})} \mathrm{u}_{\mathrm{i}}+\mathrm{N}_{\mathrm{j}}^{(\mathrm{e})} \mathrm{u}_{\mathrm{j}}$

$\mathrm{v}^{(\mathrm{e})}=\mathrm{N}_{\mathrm{i}}^{(\mathrm{e})} \mathrm{v}_{\mathrm{i}}+\mathrm{N}_{\mathrm{j}}^{(\mathrm{e})} \mathrm{v}_{\mathrm{j}}$ 
where $\mathrm{N}_{\mathrm{i}}^{(\mathrm{e})}$ and $\mathrm{N}_{\mathrm{j}}^{(\mathrm{e})}$ are pyramid functions:

$\mathrm{N}_{\mathrm{i}}^{(\mathrm{e})}=\frac{1}{\mathrm{~L}}\left(\mathrm{a}_{\mathrm{i}}+\mathrm{b}_{\mathrm{i}} \mathrm{x}\right)$ such that $\mathrm{a}_{\mathrm{i}}=\mathrm{x}_{\mathrm{j}}$ and $b_{i}=-1$

and

$\mathrm{N}_{\mathrm{j}}^{(\mathrm{e})}=\frac{1}{\mathrm{~L}}\left(\mathrm{a}_{\mathrm{j}}+\mathrm{b}_{\mathrm{j}} \mathrm{x}\right)$ such that $\mathrm{a}_{\mathrm{j}}=-\mathrm{x}_{\mathrm{i}}$ and $b_{j}=1$

and the derivative for (5) is:

$\frac{\mathrm{du}^{(\mathrm{e})}}{\mathrm{dx}}=\frac{\mathrm{dN}_{\mathrm{i}}}{\mathrm{dx}} \mathrm{u}_{\mathrm{i}}+\frac{\mathrm{dN}_{\mathrm{j}}}{\mathrm{dx}} \mathrm{u}_{\mathrm{j}}$

$\frac{d v^{(e)}}{d x}=\frac{d N_{i}}{d x} v_{i}+\frac{d N_{j}}{d x} v_{j}$

Also integral over the element must involve the integrals of the pyramid functions[1]:

$\int_{\mathrm{L}} \mathrm{N}_{\mathrm{i}} \mathrm{dx}=\int_{\mathrm{L}} \mathrm{N}_{\mathrm{j}} \mathrm{dx}=\frac{1}{2} \mathrm{~L}$

thus

$\int_{\mathrm{L}} \mathrm{u}^{(\mathrm{e})} \mathrm{dx}=\frac{1}{2} \mathrm{~L}\left(\mathrm{u}_{\mathrm{i}}+\mathrm{u}_{\mathrm{j}}\right)$

$\int_{\mathrm{L}} \mathrm{v}^{(\mathrm{e})} \mathrm{dx}=\frac{1}{2} \mathrm{~L}\left(\mathrm{v}_{\mathrm{i}}+\mathrm{v}_{\mathrm{j}}\right)$

it has been shown that the general integration formula is [1]:

$\int_{\mathrm{L}} \mathrm{N}_{\mathrm{i}}^{\alpha} \mathrm{N}_{\mathrm{j}}^{\beta} \mathrm{dx}=\frac{\alpha ! \beta !}{(\alpha+\beta+1) !} \mathrm{L}$

Galerkin method uses the approximating functions as weighting functions:

$\mathrm{W}_{\mathrm{n}}=\mathrm{N}_{\mathrm{j}}^{(\mathrm{e})}+\mathrm{N}_{\mathrm{i}}^{(\mathrm{e}+1)}$

\section{Element residuals:}

The element residuals $R_{i}, R_{j}$ associated with the nodes i,j are [1]:

$\mathrm{R}_{\mathrm{i}}^{(\mathrm{e})}=-\int_{\mathrm{L}^{(\mathrm{e})}} \mathrm{W}_{\mathrm{i}}^{(\mathrm{e})} \mathrm{r}^{(\mathrm{e})} \mathrm{dx}$

$\mathrm{R}_{\mathrm{j}}^{(\mathrm{e})}=-\int_{\mathrm{L}^{(\mathrm{e})}} \mathrm{W}_{\mathrm{j}}^{(\mathrm{e})} \mathrm{r}^{(\mathrm{e})} \mathrm{dx}$

where $R_{i}^{(e)}$ is contribution of element (e) to residual in equation (1) for node (i), and $R_{j}^{(e)}$ is contribution of element (e) to residual in equation (1) for node $(j)$. 
For the equation (2), the element residuals $O_{i}, O_{j}$ associated with the nodes $\mathrm{i}, \mathrm{j}$ are:

$$
\begin{aligned}
& \mathrm{O}_{\mathrm{i}}^{(\mathrm{e})}=-\int_{\mathrm{L}^{(e)}} \mathrm{W}_{\mathrm{i}}^{(\mathrm{e})} \mathrm{o}^{(\mathrm{e})} \mathrm{dx} \\
& \mathrm{O}_{\mathrm{j}}^{(\mathrm{e})}=-\int_{\mathrm{L}^{(\mathrm{e})}} \mathrm{W}_{j}^{(\mathrm{e})} \mathrm{o}^{(\mathrm{e})} \mathrm{dx}
\end{aligned}
$$

Galerkin's method [8] is employed for the finite element formulation in space and time, after substituting the approximation $u^{*}(x, t)$ and $v^{*}(x, t)$, the error for equation (1) and (2) are:

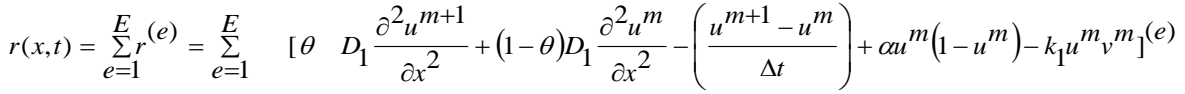

$$
\begin{aligned}
& 0(x, t)=\sum_{e=1}^{E} 0^{(e)}=\sum_{e=1}^{E} \quad\left[\theta D_{2} \frac{\partial^{2} v^{m+1}}{\partial x^{2}}+(1-\theta) D_{2} \frac{\partial^{2} v^{m}}{\partial x^{2}}-\left(\frac{v^{m+1}-v^{m}}{\Delta t}\right)-\mu v^{m}-k_{2} u^{m+1} v^{m}\right]^{(e)}
\end{aligned}
$$

$\mathrm{E}$ is the total number of elements.

for the (ith) node in element (e) in the equation (11), the residual is:

$\mathrm{R}_{\mathrm{i}}^{(\mathrm{e})}=\left[\mathrm{R}_{\mathrm{i}}\right]_{\text {space }}^{(\mathrm{e})}+\left[\mathrm{R}_{\mathrm{i}}\right]_{\text {time }}^{(\mathrm{e})}$

where

$$
\begin{aligned}
& {\left[\mathrm{R}_{\mathrm{i}}\right]_{\text {space }}^{(\mathrm{e})}=-\int_{\mathrm{L}^{(e)}}\left(\mathrm{N}_{\mathrm{i}}\left[\theta \mathrm{D}_{1} \frac{\partial^{2} \mathrm{u}^{\mathrm{m}+1}}{\partial \mathrm{x}^{2}}+(1-\theta) \mathrm{D}_{1} \frac{\partial^{2} \mathrm{u}^{\mathrm{m}}}{\partial \mathrm{x}^{2}}+\alpha \mathrm{u}^{\mathrm{m}}\left(1-\mathrm{u}^{\mathrm{m}}\right)-\mathrm{k}_{1} \mathrm{u}^{\mathrm{m}} \mathrm{v}^{\mathrm{m}}\right]\right)^{(\mathrm{e})} \mathrm{dx}} \\
& {\left[\mathrm{R}_{\mathrm{i}}\right]_{\text {time }}^{(\mathrm{e})}=-\int_{\mathrm{L}^{(e))}}\left(\mathrm{N}_{\mathrm{i}}\left[-\left(\frac{\mathrm{u}^{\mathrm{m}+1}-\mathrm{u}^{\mathrm{m}}}{\Delta \mathrm{t}}\right)\right]\right)^{(\mathrm{e})} \mathrm{dx}}
\end{aligned}
$$

also for the (jth) node in element (e) in the equation (11), the residual is: $\mathrm{R}_{\mathrm{j}}^{(\mathrm{e})}=\left[\mathrm{R}_{\mathrm{j}}\right]_{\text {space }}^{(\mathrm{e})}+\left[\mathrm{R}_{\mathrm{j}}\right]_{\text {time }}^{(\mathrm{e})}$

where

$$
\begin{aligned}
& {\left[\mathrm{R}_{\mathrm{j}}\right]_{\text {space }}^{(\mathrm{e})}=-\int_{\mathrm{L}^{(e)}}\left(\mathrm{N}_{\mathrm{j}}\left[\theta \mathrm{D}_{1} \frac{\partial^{2} \mathrm{u}^{\mathrm{m}+1}}{\partial \mathrm{x}^{2}}+(1-\theta) \mathrm{D}_{1} \frac{\partial^{2} \mathrm{u}^{\mathrm{m}}}{\partial \mathrm{x}^{2}}+\alpha \mathrm{u}^{\mathrm{m}}\left(1-\mathrm{u}^{\mathrm{m}}\right)-\mathrm{k}_{1} \mathrm{u}^{\mathrm{m}} \mathrm{v}^{\mathrm{m}}\right]\right)^{(\mathrm{e})} \mathrm{dx}} \\
& {\left[\mathrm{R}_{\mathrm{j}}\right]_{\text {time }}^{(\mathrm{e})}=-\int_{\mathrm{L}^{(e))}}\left(\mathrm{N}_{\mathrm{j}}\left[-\left(\frac{\mathrm{u}^{\mathrm{m}+1}-\mathrm{u}^{\mathrm{m}}}{\Delta \mathrm{t}}\right)\right]\right)^{(\mathrm{e})} \mathrm{dx}}
\end{aligned}
$$

for the (ith) node in element (e) in the equation (12), the residual is:

$$
\begin{aligned}
& \mathrm{O}_{\mathrm{i}}^{(\mathrm{e})}=\left[\mathrm{O}_{\mathrm{i}}\right]_{\text {space }}^{(\mathrm{e})}+\left[\mathrm{O}_{\mathrm{i}}\right]_{\text {time }}^{(\mathrm{e})} \\
& {\left[\mathrm{O}_{\mathrm{i}}\right]_{\text {space }}^{(\mathrm{e})}=-\int_{\mathrm{L}^{(\mathrm{e})}}\left(\mathrm{N}_{\mathrm{i}}\left[\theta \mathrm{D}_{2} \frac{\partial^{2} \mathrm{v}^{\mathrm{m}+1}}{\partial \mathrm{x}^{2}}+(1-\theta) \mathrm{D}_{2} \frac{\partial^{2} \mathrm{v}^{\mathrm{m}}}{\partial \mathrm{x}^{2}}-\mu \mathrm{v}^{\mathrm{m}}+\mathrm{k}_{2} \mathrm{u}^{\mathrm{m}+1} \mathrm{v}^{\mathrm{m}}\right]\right)^{(\mathrm{e})} \mathrm{dx}}
\end{aligned}
$$


$\left[\mathrm{O}_{\mathrm{i}}\right]_{\text {time }}^{(\mathrm{e})}=-\int_{\left.\mathrm{L}^{(\mathrm{e})}\right)}\left(\mathrm{N}_{\mathrm{i}}\left[-\left(\frac{\mathrm{v}^{\mathrm{m}+1}-\mathrm{v}^{\mathrm{m}}}{\Delta \mathrm{t}}\right)\right]\right)^{(\mathrm{e})} \mathrm{dx}$

also for the (jth) node in element (e) in the equation (11), the residual is:

$\mathrm{O}_{\mathrm{j}}^{(\mathrm{e})}=\left[\mathrm{O}_{\mathrm{j}}\right]_{\text {space }}^{(\mathrm{e})}+\left[\mathrm{O}_{\mathrm{j}}\right]_{\text {time }}^{(\mathrm{e})}$

where

$\left[\mathrm{O}_{\mathrm{j}}\right]_{\text {space }}^{(\mathrm{e})}=-\int_{\mathrm{L}^{(e)}}\left(\mathrm{N}_{\mathrm{j}}\left[\theta \mathrm{D}_{2} \frac{\partial^{2} \mathrm{v}^{\mathrm{m}+1}}{\partial \mathrm{x}^{2}}+(1-\theta) \mathrm{D}_{2} \frac{\partial^{2} \mathrm{v}^{\mathrm{m}}}{\partial \mathrm{x}^{2}}-\mu \mathrm{v}^{\mathrm{m}}+\mathrm{k}_{2} \mathrm{u}^{\mathrm{m}+1} \mathrm{v}^{\mathrm{m}}\right]\right)^{(\mathrm{e})} \mathrm{dx}$

$\left[\mathrm{O}_{\mathrm{j}}\right]_{\text {time }}^{(\mathrm{e})}=-\int_{\mathrm{L}^{(e))}}\left(\mathrm{N}_{\mathrm{j}}\left[-\left(\frac{\mathrm{v}^{\mathrm{m}+1}-\mathrm{v}^{\mathrm{m}}}{\Delta \mathrm{t}}\right)\right]\right)^{(\mathrm{e})} \mathrm{dx}$

Now, we use the integrating by parts and use the neumann boundary conditions(4), the equation (13) becomes:

$\left[R_{i}\right]_{\text {space }}^{(e)}=\int_{L^{(e)}}\left[\theta D_{1} \frac{d N_{i}}{d x} \frac{\partial u^{m+1}}{\partial x}+(1-\theta) D_{1} \frac{d N_{i}}{d x} \frac{\partial u^{m}}{\partial x}-N_{i}\left(\alpha u^{m}\left(1-u^{m}\right)-k_{1} u^{m} v^{m}\right)\right]^{(e)} d x$

from equations (5),(6) and (7), we get:

$$
\begin{aligned}
& R_{\mathrm{i}} \mathrm{R}_{\text {space }}^{(\mathrm{e})}=\left[\theta \mathrm{D}_{1} \frac{\mathrm{b}_{\mathrm{i}} \mathrm{b}_{\mathrm{i}}}{\mathrm{L}}\right]^{(\mathrm{e})} \mathrm{u}_{\mathrm{i}}^{\mathrm{m}+1}+\left[\theta \mathrm{D}_{1} \frac{\mathrm{b}_{\mathrm{i}} \mathrm{b}_{\mathrm{j}}}{\mathrm{L}}\right]^{(\mathrm{e})} \mathrm{u}_{\mathrm{j}}^{\mathrm{m}+1}+\left[(1-\theta) \mathrm{D}_{1} \frac{\mathrm{b}_{\mathrm{i}} \mathrm{b}_{\mathrm{i}}}{\mathrm{L}}\right]^{(\mathrm{e})} \mathrm{u}_{\mathrm{i}}^{\mathrm{m}} \\
& \quad\left[(1-\theta) \mathrm{D}_{1} \frac{\mathrm{b}_{\mathrm{i}} \mathrm{b}_{\mathrm{j}}}{\mathrm{L}}\right]^{(\mathrm{e})} \mathrm{u}_{\mathrm{j}}^{\mathrm{m}}-\alpha \int_{\mathrm{L}^{(\mathrm{e})}}\left[\mathrm{N}_{\mathrm{i}}\left(\mathrm{N}_{\mathrm{i}} \mathrm{u}_{\mathrm{i}}^{\mathrm{m}}+\mathrm{N}_{\mathrm{j}} \mathrm{u}_{\mathrm{j}}^{\mathrm{m}}\right)\right]^{(\mathrm{e})} \mathrm{dx}+\alpha \int_{\mathrm{L}^{(e)}}\left[\mathrm{N}_{\mathrm{i}}\left(\mathrm{N}_{\mathrm{i}} \mathrm{u}_{\mathrm{i}}^{\mathrm{m}}+\mathrm{N}_{\mathrm{j}} \mathrm{u}_{\mathrm{j}}^{\mathrm{m}}\right)^{2}\right]^{(\mathrm{e})} \mathrm{dx} \\
& +\mathrm{k}_{1} \int_{\mathrm{L}^{(\mathrm{e})}}\left[\mathrm{N}_{\mathrm{i}}\left(\mathrm{N}_{\mathrm{i}} \mathrm{u}_{\mathrm{i}}^{\mathrm{m}}+\mathrm{N}_{\mathrm{j}} \mathrm{u}_{\mathrm{j}}^{\mathrm{m}}\right)\left(\mathrm{N}_{\mathrm{i}} \mathrm{v}_{\mathrm{i}}^{\mathrm{m}}+\mathrm{N}_{\mathrm{j}} \mathrm{v}_{\mathrm{j}}^{\mathrm{m}}\right)\right]^{(\mathrm{e})} \mathrm{dx}
\end{aligned}
$$

and by using equation (9), the above equation becomes:

$$
\begin{aligned}
& {\left[_{i} \mathrm{R}_{\text {space }}^{(e)}\right.}=\left[\theta \mathrm{D}_{1} \frac{\mathrm{b}_{\mathrm{i}} \mathrm{b}_{\mathrm{i}}}{\mathrm{L}}\right]^{(\mathrm{e})} \mathrm{u}_{\mathrm{i}}^{\mathrm{m}+1}+\left[\theta \mathrm{D}_{1} \frac{\mathrm{b}_{\mathrm{i}} \mathrm{b}_{\mathrm{j}}}{\mathrm{L}}\right]^{(\mathrm{e})} \mathrm{u}_{\mathrm{j}}^{\mathrm{m}+1}+\left[(1-\theta) \mathrm{D}_{1} \frac{\mathrm{b}_{\mathrm{i}} \mathrm{b}_{\mathrm{i}}}{\mathrm{L}}\right]^{(\mathrm{e})} \mathrm{u}_{\mathrm{i}}^{\mathrm{m}} \\
& {\left[(1-\theta) \mathrm{D}_{1} \frac{\mathrm{b}_{\mathrm{i}} \mathrm{b}_{\mathrm{j}}}{\mathrm{L}}\right]^{(\mathrm{e})} \mathrm{u}_{\mathrm{j}}^{\mathrm{m}}-\left[\alpha \frac{\mathrm{L}}{3} \mathrm{u}_{\mathrm{i}}^{\mathrm{m}}\right]^{(\mathrm{e})}-\left[\frac{\alpha \mathrm{L}}{6} \mathrm{u}_{\mathrm{j}}^{\mathrm{m}}\right]^{(\mathrm{e})}+\left[\frac{\alpha \mathrm{L}}{4}\left(\mathrm{u}_{\mathrm{i}}^{\mathrm{m}}\right)^{2}\right]^{(\mathrm{e})}+} \\
&+\left[\frac{\alpha \mathrm{L}}{6} \mathrm{u}_{\mathrm{i}}^{\mathrm{m}} \mathrm{u}_{\mathrm{j}}^{\mathrm{m}}\right]^{(\mathrm{e})}+\left[\frac{\alpha \mathrm{L}}{12}\left(\mathrm{u}_{\mathrm{j}}^{\mathrm{m}}\right)^{2}\right]^{(\mathrm{e})}+\left[\frac{\mathrm{k}_{1} \mathrm{~L}}{4} \mathrm{u}_{\mathrm{i}}^{\mathrm{m}} \mathrm{v}_{\mathrm{i}}^{\mathrm{m}}\right]^{(\mathrm{e})}+\left[\frac{\mathrm{k}_{1} \mathrm{~L}}{12} \mathrm{u}_{\mathrm{i}}^{\mathrm{m}} \mathrm{v}_{\mathrm{j}}^{\mathrm{m}}\right]^{(\mathrm{e})} \\
&+\left[\frac{\mathrm{k}_{1} \mathrm{~L}}{12} \mathrm{u}_{\mathrm{j}}^{\mathrm{m}} \mathrm{v}_{\mathrm{i}}^{\mathrm{m}}\right]^{(\mathrm{e})}+\left[\frac{\mathrm{k}_{1} \mathrm{~L}}{12} \mathrm{u}_{\mathrm{j}}^{\mathrm{m}} \mathrm{v}_{\mathrm{j}}^{\mathrm{m}}\right]^{(\mathrm{e})}
\end{aligned}
$$

from the time term (14) in the (ith) node, we get: 


$$
\begin{aligned}
{\left[\mathrm{R}_{\mathrm{i}}\right]_{\text {time }}^{(\mathrm{e})} } & =-\int_{\mathrm{L}^{(e)}}\left(\mathrm{N}_{\mathrm{i}}\left[-\left(\frac{\mathrm{u}^{\mathrm{m}+1}-\mathrm{u}^{\mathrm{m}}}{\Delta \mathrm{t}}\right)\right]\right)^{(\mathrm{e})} \mathrm{dx} \\
& =-\int_{\mathrm{L}^{(e)}}\left(\mathrm{N}_{\mathrm{i}}\left[-\frac{1}{\Delta \mathrm{t}}\left(\mathrm{N}_{\mathrm{i}} \mathrm{u}_{\mathrm{i}}^{\mathrm{m}+1}+\mathrm{N}_{\mathrm{j}} \mathrm{u}_{\mathrm{j}}^{\mathrm{m}+1}\right)+\frac{1}{\Delta \mathrm{t}}\left(\mathrm{N}_{\mathrm{i}} \mathrm{u}_{\mathrm{i}}^{\mathrm{m}}+\mathrm{N}_{\mathrm{j}} \mathrm{u}_{\mathrm{j}}^{\mathrm{m}}\right)\right]\right)^{(\mathrm{e})} \mathrm{dx} \\
& =\left[\frac{\mathrm{L}}{6 \Delta \mathrm{t}}\left(2 \mathrm{u}_{\mathrm{i}}^{\mathrm{m}+1}+\mathrm{u}_{\mathrm{j}}^{\mathrm{m}+1}\right)-\frac{\mathrm{L}}{6 \Delta \mathrm{t}}\left(2 \mathrm{u}_{\mathrm{i}}^{\mathrm{m}}+\mathrm{u}_{\mathrm{j}}^{\mathrm{m}}\right)\right]^{(\mathrm{e})}
\end{aligned}
$$

finally this becomes:

$\left[\mathrm{R}_{\mathrm{i}}\right]_{\text {time }}^{(\mathrm{e})}=\left[\frac{\mathrm{L}}{3 \Delta \mathrm{t}}\right]^{(\mathrm{e})} \mathrm{u}_{\mathrm{i}}^{\mathrm{m}+1}+\left[\frac{\mathrm{L}}{6 \Delta \mathrm{t}}\right]^{(\mathrm{e})} \mathrm{u}_{\mathrm{j}}^{\mathrm{m}+1}-\left[\frac{\mathrm{L}}{3 \Delta \mathrm{t}}\right]^{(\mathrm{e})} \mathrm{u}_{\mathrm{i}}^{\mathrm{m}}-\left[\frac{\mathrm{L}}{6 \Delta \mathrm{t}}\right]^{(\mathrm{e})} \mathrm{u}_{\mathrm{j}}^{\mathrm{m}}$

Similarly, the equation (15) for the (jth) node in element (e) becomes:

$$
\begin{aligned}
{\left[\mathrm{R}_{\mathrm{j}}\right]_{\text {space }}^{(e)} } & =\left[\theta \mathrm{D}_{1} \frac{\mathrm{b}_{\mathrm{j}} \mathrm{b}_{\mathrm{i}}}{\mathrm{L}}\right]^{(\mathrm{e})} \mathrm{u}_{\mathrm{i}}^{\mathrm{m}+1}+\left[\theta \mathrm{D}_{1} \frac{\mathrm{b}_{\mathrm{j}} \mathrm{b}_{\mathrm{j}}}{\mathrm{L}}\right]^{(\mathrm{e})} \mathrm{u}_{\mathrm{j}}^{\mathrm{m}+1}+\left[(1-\theta) \mathrm{D}_{1} \frac{\mathrm{b}_{\mathrm{j}} \mathrm{b}_{\mathrm{i}}}{\mathrm{L}}\right]^{(\mathrm{e})} \mathrm{u}_{\mathrm{i}}^{\mathrm{m}} \\
& {\left[(1-\theta) \mathrm{D}_{1} \frac{\mathrm{b}_{\mathrm{j}} \mathrm{b}_{\mathrm{j}}}{\mathrm{L}}\right]^{(\mathrm{e})} \mathrm{u}_{\mathrm{j}}^{\mathrm{m}}-\left[\alpha \frac{\mathrm{L}}{6} \mathrm{u}_{\mathrm{i}}^{\mathrm{m}}\right]^{(\mathrm{e})}-\left[\frac{\alpha \mathrm{L}}{3} \mathrm{u}_{\mathrm{j}}^{\mathrm{m}}\right]^{(\mathrm{e})}+\left[\frac{\alpha \mathrm{L}}{12}\left(\mathrm{u}_{\mathrm{i}}^{\mathrm{m}}\right)^{2}\right]^{(\mathrm{e})}+} \\
+ & {\left[\frac{\alpha \mathrm{L}}{6} \mathrm{u}_{\mathrm{i}}^{\mathrm{m}} \mathrm{u}_{\mathrm{j}}^{\mathrm{m}}\right]^{(\mathrm{e})}+\left[\frac{\alpha \mathrm{L}}{4}\left(\mathrm{u}_{\mathrm{j}}^{\mathrm{m}}\right)^{2}\right]^{(\mathrm{e})}+\left[\frac{\mathrm{k}_{1} \mathrm{~L}}{12} \mathrm{u}_{\mathrm{i}}^{\mathrm{m}} \mathrm{v}_{\mathrm{i}}^{\mathrm{m}}\right]^{(\mathrm{e})}+\left[\frac{\mathrm{k}_{1} \mathrm{~L}}{12} \mathrm{u}_{\mathrm{i}}^{\mathrm{m}} \mathrm{v}_{\mathrm{j}}^{\mathrm{m}}\right]^{(\mathrm{e})} } \\
+ & {\left[\frac{\mathrm{k}_{1} \mathrm{~L}}{12} \mathrm{u}_{\mathrm{j}}^{\mathrm{m}} \mathrm{v}_{\mathrm{i}}^{\mathrm{m}}\right]^{(\mathrm{e})}+\left[\frac{\mathrm{k}_{1} \mathrm{~L}}{4} \mathrm{u}_{\mathrm{j}}^{\mathrm{m}} \mathrm{v}_{\mathrm{j}}^{\mathrm{m}}\right]^{(\mathrm{e})} }
\end{aligned}
$$

by the same way as in residual (ith), we get:

$$
\begin{aligned}
{\left[R_{j}\right]_{\text {time }}^{(e)} } & =-\int_{L^{(e)}}\left(N_{j}\left[-\left(\frac{u^{m+1}-u^{m}}{\Delta t}\right)\right]\right)^{(e)} d x \\
& =\left[\frac{L}{6 \Delta t}\right]^{(e)} u_{i}^{m+1}+\left[\frac{L}{3 \Delta t}\right]^{(e)} u_{j}^{m+1}-\left[\frac{L}{6 \Delta t}\right]^{(e)} u_{i}^{m}-\left[\frac{L}{3 \Delta t}\right]^{(e)} u_{j}^{m}
\end{aligned}
$$

this completes all of the residuals of the equation (11).

For equation (12) and by the same mannar we have:

$$
\left[\mathrm{O}_{\mathrm{i}}\right]_{\text {space }}^{(\mathrm{e})}=\int_{\mathrm{L}^{(e)}}\left[\theta \mathrm{D}_{2} \frac{\mathrm{dN}}{\mathrm{dx}} \frac{\partial \mathrm{v}^{\mathrm{m}+1}}{\partial \mathrm{x}}+(1-\theta) \mathrm{D}_{2} \frac{\mathrm{dN}}{\mathrm{dx}} \frac{\partial \mathrm{v}^{\mathrm{m}}}{\partial \mathrm{x}}+\mathrm{N}_{\mathrm{i}}\left(\mu \mathrm{v}^{\mathrm{m}}-\mathrm{k}_{2} \mathrm{u}^{\mathrm{m}+1} \mathrm{v}^{\mathrm{m}}\right)\right]^{(\mathrm{e})} \mathrm{dx}
$$

by using the equations (5),(6),(7) and (9), we get: 


$$
\begin{aligned}
{\left[\mathrm{O}_{\mathrm{i}}\right]_{\text {space }}^{(\mathrm{e})} } & =\left[\theta \mathrm{D}_{2} \frac{\mathrm{b}_{\mathrm{i}} \mathrm{b}_{\mathrm{i}}}{\mathrm{L}}\right]^{(\mathrm{e})} \mathrm{v}_{\mathrm{i}}^{\mathrm{m}+1}+\left[\theta \mathrm{D}_{2} \frac{\mathrm{b}_{\mathrm{i}} \mathrm{b}_{\mathrm{j}}}{\mathrm{L}}\right]^{(\mathrm{e})} \mathrm{v}_{\mathrm{j}}^{\mathrm{m}+1}+\left[(1-\theta) \mathrm{D}_{2} \frac{\mathrm{b}_{\mathrm{i}} \mathrm{b}_{\mathrm{i}}}{\mathrm{L}}\right]^{(\mathrm{e})} \mathrm{v}_{\mathrm{i}}^{\mathrm{m}} \\
& {\left[(1-\theta) \mathrm{D}_{2} \frac{\mathrm{b}_{\mathrm{i}} \mathrm{b}_{\mathrm{j}}}{\mathrm{L}}\right]^{(\mathrm{e})} \mathrm{v}_{\mathrm{j}}^{\mathrm{m}}+\left[\frac{\mu \mathrm{L}}{3} \mathrm{v}_{\mathrm{i}}^{\mathrm{m}}\right]^{(\mathrm{e})}+\left[\frac{\mu \mathrm{L}}{6} \mathrm{v}_{\mathrm{j}}^{\mathrm{m}}\right]^{(\mathrm{e})}-\left[\frac{\mathrm{k}_{2} \mathrm{~L}}{4} \mathrm{u}_{\mathrm{i}}^{\mathrm{m}+1} \mathrm{v}_{\mathrm{i}}^{\mathrm{m}}\right]^{(\mathrm{e})} } \\
& -\left[\frac{\mathrm{k}_{2} \mathrm{~L}}{12} \mathrm{u}_{\mathrm{i}}^{\mathrm{m}+1} \mathrm{v}_{\mathrm{j}}^{\mathrm{m}}\right]^{(\mathrm{e})}-\left[\frac{\mathrm{k}_{2} \mathrm{~L}}{12} \mathrm{u}_{\mathrm{j}}^{\mathrm{m}+1} \mathrm{v}_{\mathrm{i}}^{\mathrm{m}}\right]^{(\mathrm{e})}-\left[\frac{\mathrm{k}_{2} \mathrm{~L}}{12} \mathrm{u}_{\mathrm{j}}^{\mathrm{m}+1} \mathrm{v}_{\mathrm{j}}^{\mathrm{m}}\right]^{(\mathrm{e})}
\end{aligned}
$$

the time term (18) in the (ith) node is:

$$
\begin{aligned}
{\left[\mathrm{O}_{\mathrm{i}}\right]_{\text {time }}^{(\mathrm{e})} } & =-\int_{\left.\mathrm{L}^{(e)}\right)}\left(\mathrm{N}_{\mathrm{i}}\left[-\left(\frac{\mathrm{v}^{\mathrm{m}+1}-\mathrm{v}^{\mathrm{m}}}{\Delta \mathrm{t}}\right)\right]\right)^{(\mathrm{e})} \mathrm{dx} \\
& =\left[\frac{\mathrm{L}}{3 \Delta \mathrm{t}}\right]^{(\mathrm{e})} \mathrm{v}_{\mathrm{i}}^{\mathrm{m}+1}+\left[\frac{\mathrm{L}}{6 \Delta \mathrm{t}}\right]^{(\mathrm{e})} \mathrm{v}_{\mathrm{j}}^{\mathrm{m}+1}-\left[\frac{\mathrm{L}}{3 \Delta \mathrm{t}}\right]^{(\mathrm{e})} \mathrm{v}_{\mathrm{i}}^{\mathrm{m}}-\left[\frac{\mathrm{L}}{6 \Delta \mathrm{t}}\right]^{(\mathrm{e})} \mathrm{v}_{\mathrm{j}}^{\mathrm{m}}
\end{aligned}
$$

also for the (jth) node in element (e), the equation (19) becomes:

$$
\begin{aligned}
{\left[\mathrm{O}_{\mathrm{j}}\right]_{\text {space }}^{(e)} } & =\left[\theta \mathrm{D}_{2} \frac{\mathrm{b}_{\mathrm{i}} \mathrm{b}_{\mathrm{i}}}{\mathrm{L}}\right]^{(\mathrm{e})} \mathrm{v}_{\mathrm{i}}^{\mathrm{m}+1}+\left[\theta \mathrm{D}_{2} \frac{\mathrm{b}_{\mathrm{i}} \mathrm{b}_{\mathrm{j}}}{\mathrm{L}}\right]^{(\mathrm{e})} \mathrm{v}_{\mathrm{j}}^{\mathrm{m}+1}+\left[(1-\theta) \mathrm{D}_{2} \frac{\mathrm{b}_{\mathrm{i}} \mathrm{b}_{\mathrm{i}}}{\mathrm{L}}\right]^{(\mathrm{e})} \mathrm{v}_{\mathrm{i}}^{\mathrm{m}} \\
& {\left[(1-\theta) \mathrm{D}_{2} \frac{\mathrm{b}_{\mathrm{i}} \mathrm{b}_{\mathrm{j}}}{\mathrm{L}}\right]^{(\mathrm{e})} \mathrm{v}_{\mathrm{j}}^{\mathrm{m}}+\left[\frac{\mu \mathrm{L}}{6} \mathrm{v}_{\mathrm{i}}^{\mathrm{m}}\right]^{(\mathrm{e})}+\left[\frac{\mu \mathrm{L}}{3} \mathrm{v}_{\mathrm{j}}^{\mathrm{m}}\right]^{(\mathrm{e})}-\left[\frac{\mathrm{k}_{2} \mathrm{~L}}{12} \mathrm{u}_{\mathrm{i}}^{\mathrm{m}+1} \mathrm{v}_{\mathrm{i}}^{\mathrm{m}}\right]^{(\mathrm{e})} } \\
& -\left[\frac{\mathrm{k}_{2} \mathrm{~L}}{12} \mathrm{u}_{\mathrm{i}}^{\mathrm{m}+1} \mathrm{v}_{\mathrm{j}}^{\mathrm{m}}\right]^{(\mathrm{e})}-\left[\frac{\mathrm{k}_{2} \mathrm{~L}}{12} \mathrm{u}_{\mathrm{j}}^{\mathrm{m}+1} \mathrm{v}_{\mathrm{i}}^{\mathrm{m}}\right]^{(\mathrm{e})}-\left[\frac{\mathrm{k}_{2} \mathrm{~L}}{4} \mathrm{u}_{\mathrm{j}}^{\mathrm{m}+1} \mathrm{v}_{\mathrm{j}}^{\mathrm{m}}\right]^{(\mathrm{e})}
\end{aligned}
$$

by the same way as in residual (ith), we get:

$$
\begin{aligned}
{\left[\mathrm{O}_{\mathrm{j}}\right]_{\text {time }}^{(\mathrm{e})} } & =-\int_{\mathrm{L}^{(e))}}\left(\mathrm{N}_{\mathrm{j}}\left[-\left(\frac{\mathrm{v}^{\mathrm{m}+1}-\mathrm{v}^{\mathrm{m}}}{\Delta \mathrm{t}}\right)\right]\right)^{(\mathrm{e})} \mathrm{dx} \\
& =\left[\frac{\mathrm{L}}{6 \Delta \mathrm{t}}\right]^{(\mathrm{e})} \mathrm{v}_{\mathrm{i}}^{\mathrm{m}+1}+\left[\frac{\mathrm{L}}{3 \Delta \mathrm{t}}\right]^{(\mathrm{e})} \mathrm{v}_{\mathrm{j}}^{\mathrm{m}+1}-\left[\frac{\mathrm{L}}{6 \Delta \mathrm{t}}\right]^{(\mathrm{e})} \mathrm{v}_{\mathrm{i}}^{\mathrm{m}}-\left[\frac{\mathrm{L}}{3 \Delta \mathrm{t}}\right]^{(\mathrm{e})} \mathrm{v}_{\mathrm{j}}^{\mathrm{m}}
\end{aligned}
$$

\section{Element matrices:}

It is convenient to organize the residauls in element matrix form because a set of simultaneous nodal equations must be solved at each time step. Then the normal assembly procedure can be used. The terms involving nodal values at time step $(m+1)$ are unknown and appear in the element matrix. All other terms are known and go in the element column [1].

They have the form:

$$
\left[\begin{array}{l}
R_{i} \\
R_{j}
\end{array}\right]^{(e)}=[B]^{(e)}\left[\begin{array}{l}
u_{i}^{m+1} \\
u_{j}^{m+1}
\end{array}\right]-[C]^{(e)}\left[\begin{array}{l}
u_{i}^{m} \\
u_{j}^{m}
\end{array}\right]
$$


the particular components can now be evaluated.

The element residuals are written as:

$$
\left[\begin{array}{l}
R_{i} \\
R_{j}
\end{array}\right]^{(e)}=\left[\begin{array}{l}
R_{i} \\
R_{j}
\end{array}\right]_{\text {space }}^{(e)}+\left[\begin{array}{l}
R_{i} \\
R_{j}
\end{array}\right]_{\text {time }}^{(e)}
$$

substituting the equation (22) and (24) for the space residual yields:

$$
\begin{aligned}
& {\left[\begin{array}{l}
R_{i} \\
R_{j}
\end{array}\right]_{\text {space }}^{(e)}=\theta D_{1}\left[\begin{array}{cc}
\frac{b_{i} b_{i}}{L} & \frac{b_{i} b_{j}}{L} \\
\frac{b_{i} b_{j}}{L} & \frac{b_{j} b_{j}}{L}
\end{array}\right]^{(e)}\left[\begin{array}{l}
u_{i}^{m+1} \\
u_{j}^{m+1}
\end{array}\right]-(1-\theta) D_{1}\left[\begin{array}{cc}
\frac{b_{i} b_{i}}{L} & \frac{b_{i} b_{j}}{L} \\
\frac{b_{i} b_{j}}{L} & \frac{b_{j} b_{j}}{L}
\end{array}\right]^{(e)}\left[\begin{array}{l}
u_{i}^{m} \\
u_{j}^{m}
\end{array}\right]} \\
& +\left[\begin{array}{cc}
-\frac{\alpha L}{3}+\frac{\alpha L}{4} u_{i}^{m}+\frac{\alpha L}{6} u_{j}^{m}+\frac{k_{1} L}{4} v_{i}^{m}+\frac{k_{1} L}{12} v_{j}^{m} & -\frac{\alpha L}{6}+\frac{\alpha L}{12} u_{j}^{m}++\frac{k_{1} L}{12} v_{i}^{m}+\frac{k_{1} L}{12} v_{j}^{m} \\
-\frac{\alpha L}{6}+\frac{\alpha L}{12} u_{i}^{m}+\frac{k_{1} L}{12} v_{i}^{m}+\frac{k_{1} L}{12} v_{j}^{m} & -\frac{\alpha L}{3}+\frac{\alpha L}{6} u_{i}^{m}+\frac{\alpha L}{4} u_{j}^{m}+\frac{k_{1} L}{12} v_{i}^{m}+\frac{k_{1} L}{4} v_{j}^{m}
\end{array}\right]^{(e)}\left[\begin{array}{c}
u_{i}^{m} \\
u_{j}^{m}
\end{array}\right]
\end{aligned}
$$

and the time residuals (23) and (25) gives:

$$
\left[\begin{array}{l}
\mathrm{R}_{\mathrm{i}} \\
\mathrm{R}_{\mathrm{j}}
\end{array}\right]_{\text {time }}^{(\mathrm{e})}=\left[\begin{array}{cc}
\frac{\mathrm{L}}{3 \Delta \mathrm{t}} & \frac{\mathrm{L}}{6 \Delta \mathrm{t}} \\
\frac{\mathrm{L}}{6 \Delta \mathrm{t}} & \frac{\mathrm{L}}{3 \Delta \mathrm{t}}
\end{array}\right]^{(\mathrm{e})}\left[\begin{array}{l}
\mathrm{u}_{\mathrm{i}}^{\mathrm{m}+1} \\
\mathrm{u}_{\mathrm{j}+1}^{\mathrm{m}+1}
\end{array}\right]+\left[\begin{array}{cc}
-\frac{\mathrm{L}}{3 \Delta \mathrm{t}} & -\frac{\mathrm{L}}{6 \Delta \mathrm{t}} \\
-\frac{\mathrm{L}}{6 \Delta \mathrm{t}} & -\frac{\mathrm{L}}{3 \Delta \mathrm{t}}
\end{array}\right]^{(\mathrm{e})}\left[\begin{array}{l}
\mathrm{u}_{\mathrm{i}}^{\mathrm{m}} \\
\mathrm{u}_{\mathrm{j}}^{\mathrm{m}}
\end{array}\right]
$$

each has a set of terms evaluated at time step $(m+1)$ and another at time step (m).

combining the terms at time step $m+1$ gives the element matrix:

$$
[B]^{(e)}=\theta\left(\frac{D_{1}}{L}\right)^{(e)}\left[\begin{array}{cc}
1 & -1 \\
-1 & 1
\end{array}\right]\left[\begin{array}{l}
u_{i}^{m+1} \\
u_{j}^{m+1}
\end{array}\right]+\left(\frac{L}{6 \Delta t}\right)^{(e)}\left[\begin{array}{ll}
2 & 1 \\
1 & 2
\end{array}\right]\left[\begin{array}{l}
u_{i}^{m+1} \\
u_{j}^{m+1}
\end{array}\right]
$$

also the element column is:

$$
\begin{aligned}
& {[C]^{(e)}=-(1-\theta)\left(\frac{D_{1}}{L}\right)^{(e)}\left[\begin{array}{cc}
1 & -1 \\
-1 & 1
\end{array}\right]\left[\begin{array}{l}
u_{i}^{m} \\
u_{j}^{m}
\end{array}\right]+\left(\frac{L}{6 \Delta t}\right)^{(e)}\left[\begin{array}{cc}
2 & 1 \\
1 & 2
\end{array}\right]^{(e)}\left[\begin{array}{c}
u_{i}^{m} \\
u_{j}^{m}
\end{array}\right] \quad . .(34)} \\
& -\left[\begin{array}{cc}
-\frac{\alpha L}{3}+\frac{\alpha L}{4} u_{i}^{m}+\frac{\alpha L}{6} u_{j}^{m}+\frac{k_{1} L}{4} v_{i}^{m}+\frac{k_{1} L}{12} v_{j}^{m} & -\frac{\alpha L}{6}+\frac{\alpha L}{12} u_{j}^{m}+\frac{k_{1} L}{12} v_{i}^{m}+\frac{k_{1} L}{12} v_{j}^{m} \\
-\frac{\alpha L}{6}+\frac{\alpha L}{12} u_{i}^{m}+\frac{k_{1} L}{12} v_{i}^{m}+\frac{k_{1} L}{12} v_{j}^{m} & -\frac{\alpha L}{3}+\frac{\alpha L}{6} u_{i}^{m}+\frac{\alpha L}{4} u_{j}^{m}+\frac{k_{1} L}{12} v_{i}^{m}+\frac{k_{1} L}{4} v_{j}^{m}
\end{array}\right]^{(e)}\left[\begin{array}{c}
u_{i}^{m} \\
u_{j}^{m}
\end{array}\right]
\end{aligned}
$$

also we can write the equations (27),(28),(29) and (30) as: 
$\left[\begin{array}{l}\mathrm{O}_{i} \\ \mathrm{O}_{j}\end{array}\right]^{(\mathrm{e})}=[\mathrm{B}]^{(e)}\left[\begin{array}{c}\mathrm{v}_{\mathrm{i}}^{\mathrm{m}+1} \\ \mathrm{v}_{\mathrm{j}}^{\mathrm{m}+1}\end{array}\right]-[\mathrm{C}]^{(\mathrm{e})}\left[\begin{array}{c}\mathrm{v}_{\mathrm{i}}^{\mathrm{m}} \\ \mathrm{v}_{\mathrm{j}}^{\mathrm{m}}\end{array}\right]$

where

$[B]^{(e)}=\theta\left(\frac{D_{2}}{L}\right)^{(e)}\left[\begin{array}{cc}1 & -1 \\ -1 & 1\end{array}\right]\left[\begin{array}{c}v_{i}^{m+1} \\ v_{j}^{m+1}\end{array}\right]+\left(\frac{L}{6 \Delta t}\right)^{(e)}\left[\begin{array}{ll}2 & 1 \\ 1 & 2\end{array}\right]\left[\begin{array}{c}v_{i}^{m+1} \\ v_{j}^{m+1}\end{array}\right]$

also the element column is:

$[C]^{(e)}=-(1-\theta)\left(\frac{D_{2}}{L}\right)^{(e)}\left[\begin{array}{cc}1 & -1 \\ -1 & 1\end{array}\right]\left[\begin{array}{c}v_{i}^{m} \\ v_{j}^{m}\end{array}\right]+\left(\frac{L}{6 \Delta t}\right)^{(e)}\left[\begin{array}{cc}2 & 1 \\ 1 & 2\end{array}\right]^{(e)}\left[\begin{array}{c}v_{i}^{m} \\ v_{j}^{m}\end{array}\right]$
$-\left[\begin{array}{c}\frac{\mu L}{3}-\frac{k_{2} L}{4} u_{i}^{m+1}-\frac{k_{2} L}{12} u_{j}^{m+1} \frac{\mu L}{6}-\frac{k_{2} L}{12} u_{i}^{m+1}-\frac{k_{2} L}{12} u_{j}^{m+1} \\ \frac{\mu L}{6}-\frac{k_{2} L}{12} u_{i}^{m+1}-\frac{k_{2} L}{12} u_{j}^{m+1} \frac{\mu L}{3}-\frac{k_{2} L}{12} u_{i}^{m+1}-\frac{k_{2} L}{4} u_{j}^{m+1}\end{array}\right]^{(e)}\left[\begin{array}{c}v_{i}^{m} \\ v_{j}^{m}\end{array}\right]$

assembling the global matrices for the equations (33) and (34), we get: 


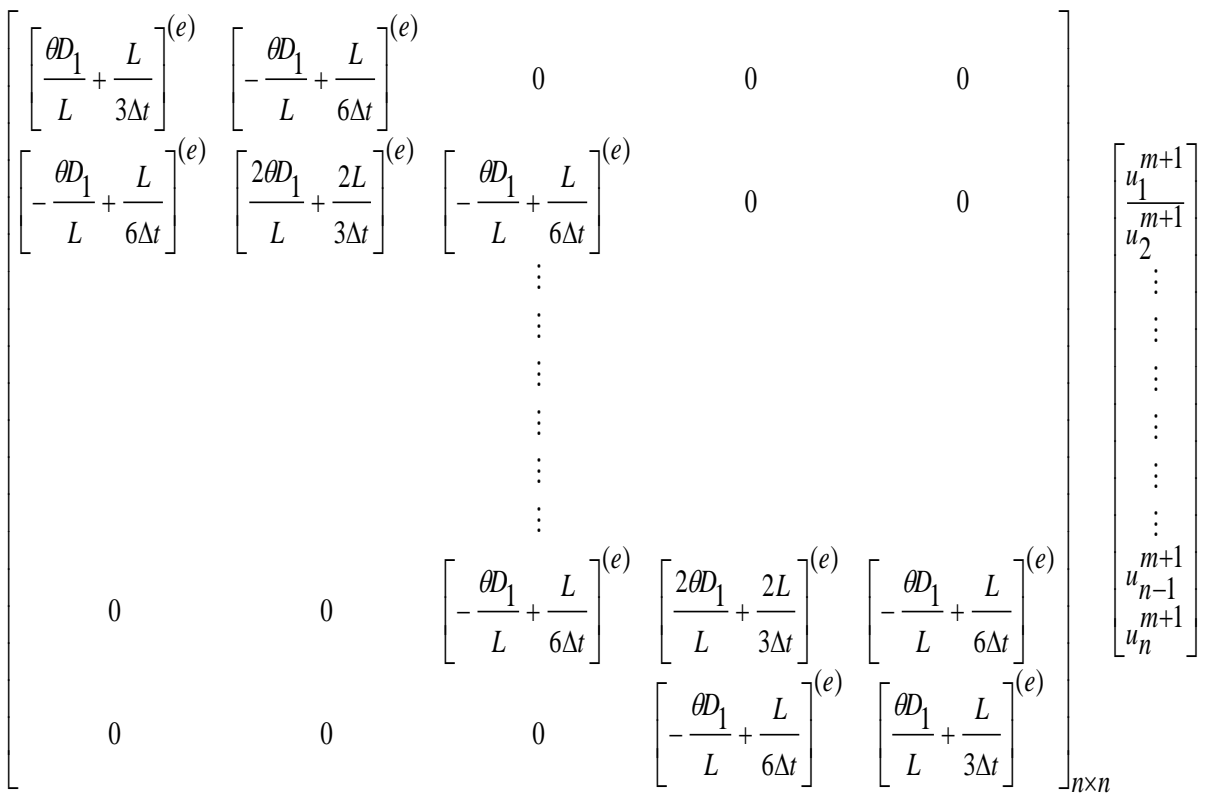

$$
\begin{aligned}
& =\left[\begin{array}{c}
a_{11} \\
a_{21} \\
a_{31} \\
\vdots \\
\vdots \\
\vdots \\
\vdots \\
\vdots \\
a_{(n-1) 1} \\
a_{n 1}
\end{array}\right]
\end{aligned}
$$

where

$$
\begin{gathered}
\mathrm{a}_{11}=\left(\frac{-(1-\theta) \mathrm{D}_{1}}{\mathrm{~L}}+\frac{2 \mathrm{~L}}{6 \Delta \mathrm{t}}+\frac{\alpha \mathrm{L}}{3}-\frac{\alpha \mathrm{L}}{4} \mathrm{u}_{1}^{\mathrm{m}}-\frac{\alpha \mathrm{L}}{6} \mathrm{u}_{2}^{\mathrm{m}}-\frac{\mathrm{k}_{1} \mathrm{~L}}{4} \mathrm{v}_{1}^{\mathrm{m}}-\frac{\mathrm{k}_{1} \mathrm{~L}}{12} \mathrm{v}_{2}^{\mathrm{m}}\right)^{(\mathrm{e})} \mathrm{u}_{1}^{\mathrm{m}} \\
+\left(\frac{(1-\theta) \mathrm{D}_{1}}{\mathrm{~L}}+\frac{\mathrm{L}}{6 \Delta \mathrm{t}}+\frac{\alpha \mathrm{L}}{6}-\frac{\alpha \mathrm{L}}{12} \mathrm{u}_{2}^{\mathrm{m}}-\frac{\mathrm{k}_{1} \mathrm{~L}}{12} \mathrm{v}_{1}^{\mathrm{m}}-\frac{\mathrm{k}_{1} \mathrm{~L}}{12} \mathrm{v}_{2}^{\mathrm{m}}\right)^{(\mathrm{e})} \mathrm{u}_{2}^{\mathrm{m}}
\end{gathered}
$$




$$
\begin{aligned}
& \mathrm{a}_{21}=\left(\frac{(1-\theta) \mathrm{D}_{1}}{\mathrm{~L}}+\frac{\mathrm{L}}{6 \Delta \mathrm{t}}+\frac{\alpha \mathrm{L}}{6}-\frac{\alpha \mathrm{L}}{12} \mathrm{u}_{1}^{\mathrm{m}}-\frac{\mathrm{k}_{1} \mathrm{~L}}{12} \mathrm{v}_{1}^{\mathrm{m}}-\frac{\mathrm{k}_{1} \mathrm{~L}}{12} \mathrm{v}_{2}^{\mathrm{m}}\right)^{(\mathrm{e})} \mathrm{u}_{1}^{\mathrm{m}} \\
& +\left(\frac{-(1-\theta) \mathrm{D}_{1}}{\mathrm{~L}}+\frac{2 \mathrm{~L}}{6 \Delta \mathrm{t}}+\frac{\alpha \mathrm{L}}{3}-\frac{\alpha \mathrm{L}}{6} \mathrm{u}_{1}^{\mathrm{m}}-\frac{\alpha \mathrm{L}}{4} \mathrm{u}_{2}^{\mathrm{m}}-\frac{\mathrm{k}_{1} \mathrm{~L}}{12} \mathrm{v}_{1}^{\mathrm{m}}-\frac{\mathrm{k}_{1} \mathrm{~L}}{4} \mathrm{v}_{2}^{\mathrm{m}}\right)^{(\mathrm{e})} \mathrm{u}_{2}^{\mathrm{m}} \\
& +\left(\frac{-(1-\theta) \mathrm{D}_{1}}{\mathrm{~L}}+\frac{2 \mathrm{~L}}{6 \Delta \mathrm{t}}+\frac{\alpha \mathrm{L}}{3}-\frac{\alpha \mathrm{L}}{6} \mathrm{u}_{3}^{\mathrm{m}}-\frac{\alpha \mathrm{L}}{4} \mathrm{u}_{2}^{\mathrm{m}}-\frac{\mathrm{k}_{1} \mathrm{~L}}{4} \mathrm{v}_{2}^{\mathrm{m}}-\frac{\mathrm{k}_{1} \mathrm{~L}}{12} \mathrm{v}_{3}^{\mathrm{m}}\right)^{(\mathrm{e})} \mathrm{u}_{2}^{\mathrm{m}} \\
& +\left(\frac{(1-\theta) \mathrm{D}_{1}}{\mathrm{~L}}+\frac{\mathrm{L}}{6 \Delta \mathrm{t}}+\frac{\alpha \mathrm{L}}{6}-\frac{\alpha \mathrm{L}}{12} \mathrm{u}_{3}^{\mathrm{m}}-\frac{\mathrm{k}_{1} \mathrm{~L}}{12} \mathrm{v}_{2}^{\mathrm{m}}-\frac{\mathrm{k}_{1} \mathrm{~L}}{12} \mathrm{v}_{3}^{\mathrm{m}}\right)^{(\mathrm{e})} \mathrm{u}_{3}^{\mathrm{m}} \\
& \mathrm{a}_{(\mathrm{n}-1) 1}=\left(\frac{(1-\theta) \mathrm{D}_{1}}{\mathrm{~L}}+\frac{\mathrm{L}}{6 \Delta \mathrm{t}}+\frac{\alpha \mathrm{L}}{6}-\frac{\alpha \mathrm{L}}{12} \mathrm{u}_{\mathrm{n}-2}^{\mathrm{m}}-\frac{\mathrm{k}_{1} \mathrm{~L}}{12} \mathrm{v}_{\mathrm{n}-2}^{\mathrm{m}}-\frac{\mathrm{k}_{1} \mathrm{~L}}{12} \mathrm{v}_{\mathrm{n}-1}^{\mathrm{m}}\right)^{(\mathrm{e})} \mathrm{u}_{\mathrm{n}-2}^{\mathrm{m}} \\
& +\left(\frac{-(1-\theta) D_{1}}{L}+\frac{2 L}{6 \Delta t}+\frac{\alpha L}{3}-\frac{\alpha L}{6} u_{n-2}^{m}-\frac{\alpha L}{4} u_{n-1}^{m}-\frac{k_{1} L}{12} v_{n-2}^{m}-\frac{k_{1} L}{4} v_{n-1}^{m}\right)^{(e)} u_{n-1}^{m} \\
& +\left(\frac{-(1-\theta) D_{1}}{\mathrm{~L}}+\frac{2 \mathrm{~L}}{6 \Delta \mathrm{t}}+\frac{\alpha \mathrm{L}}{3}-\frac{\alpha \mathrm{L}}{6} \mathrm{u}_{\mathrm{n}}^{\mathrm{m}}-\frac{\alpha \mathrm{L}}{4} \mathrm{u}_{\mathrm{n}-1}^{\mathrm{m}}-\frac{\mathrm{k}_{1} \mathrm{~L}}{4} \mathrm{v}_{\mathrm{n}-1}^{\mathrm{m}}-\frac{\mathrm{k}_{1} \mathrm{~L}}{12} \mathrm{v}_{\mathrm{n}}^{\mathrm{m}}\right)^{(\mathrm{e})} \mathrm{u}_{\mathrm{n}-1}^{\mathrm{m}} \\
& +\left(\frac{(1-\theta) D_{1}}{L}+\frac{L}{6 \Delta t}+\frac{\alpha L}{6}-\frac{\alpha L}{12} u_{n}^{m}-\frac{k_{1} L}{12} v_{n-1}^{m}-\frac{k_{1} L}{12} v_{n}^{m}\right)^{(e)} u_{n}^{m} \\
& a_{n 1}=\left(\frac{(1-\theta) D_{1}}{L}+\frac{L}{6 \Delta t}+\frac{\alpha L}{6}-\frac{\alpha L}{12} u_{n-1}^{m}-\frac{k_{1} L}{12} v_{n-1}^{m}-\frac{k_{1} L}{12} v_{n}^{m}\right)^{(e)} u_{n-1}^{m} \\
& \left(\frac{-(1-\theta) D_{1}}{L}+\frac{2 L}{6 \Delta t}+\frac{\alpha L}{3}-\frac{\alpha L}{6} u_{n-1}^{m}-\frac{\alpha L}{4} u_{n}^{m}-\frac{k_{1} L}{12} v_{n-1}^{m}-\frac{k_{1} L}{4} v_{n}^{m}\right)^{(e)} u_{n}^{m}
\end{aligned}
$$

assembling the global matrices for the equations (35) and (36) we get: 


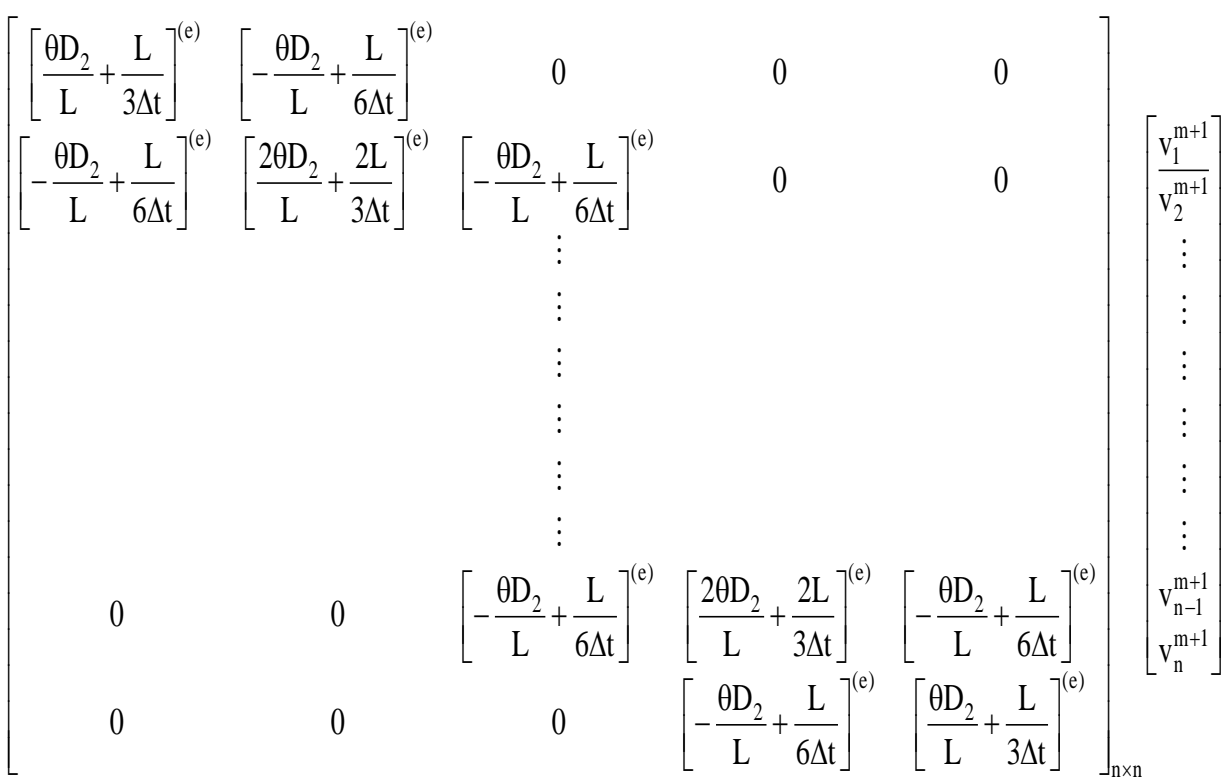

$$
\begin{aligned}
& =\left[\begin{array}{c}
b_{11} \\
b_{21} \\
b_{31} \\
\vdots \\
\vdots \\
\vdots \\
\vdots \\
\vdots \\
b_{(n-1) 1} \\
b_{n 1}
\end{array}\right]
\end{aligned}
$$

where

$$
\begin{aligned}
b_{11}= & \left(\frac{-(1-\theta) D_{2}}{L}+\frac{2 L}{6 \Delta t}-\frac{\mu L}{3}+\frac{k_{2} L}{4} u_{1}^{m+1}+\frac{k_{2} L}{12} u_{2}^{m+1}\right)^{(e)} v_{1}^{m} \\
& +\left(\frac{(1-\theta) D_{2}}{L}+\frac{L}{6 \Delta t}-\frac{\mu L}{6}+\frac{k_{2} L}{12} u_{1}^{m+1}+\frac{k_{2} L}{12} u_{2}^{m+1}\right)^{(e)} v_{2}^{m}
\end{aligned}
$$




$$
\begin{aligned}
& \mathrm{b}_{21}=\left(\frac{(1-\theta) \mathrm{D}_{2}}{\mathrm{~L}}+\frac{\mathrm{L}}{6 \Delta \mathrm{t}}-\frac{\mu \mathrm{L}}{6}+\frac{\mathrm{k}_{2} \mathrm{~L}}{12} \mathrm{u}_{1}^{\mathrm{m}+1}+\frac{\mathrm{k}_{2} \mathrm{~L}}{12} \mathrm{u}_{2}^{\mathrm{m}+1}\right)^{(\mathrm{e})} \mathrm{v}_{1}^{\mathrm{m}} \\
& +\left(\frac{-(1-\theta) \mathrm{D}_{2}}{\mathrm{~L}}+\frac{2 \mathrm{~L}}{6 \Delta \mathrm{t}}-\frac{\mu \mathrm{L}}{3}+\frac{\mathrm{k}_{2} \mathrm{~L}}{12} \mathrm{u}_{1}^{\mathrm{m}+1}+\frac{\mathrm{k}_{2} \mathrm{~L}}{4} \mathrm{u}_{2}^{\mathrm{m}+1}\right)^{(\mathrm{e})} \mathrm{v}_{2}^{\mathrm{m}} \\
& +\left(\frac{-(1-\theta) \mathrm{D}_{2}}{\mathrm{~L}}+\frac{2 \mathrm{~L}}{6 \Delta \mathrm{t}}-\frac{\mu \mathrm{L}}{3}+\frac{\mathrm{k}_{2} \mathrm{~L}}{4} \mathrm{u}_{2}^{\mathrm{m}+1}+\frac{\mathrm{k}_{2} \mathrm{~L}}{12} \mathrm{u}_{3}^{\mathrm{m}+1}\right)^{(\mathrm{e})} \mathrm{v}_{2}^{\mathrm{m}} \\
& +\left(\frac{(1-\theta) \mathrm{D}_{2}}{\mathrm{~L}}+\frac{\mathrm{L}}{6 \Delta \mathrm{t}}-\frac{\mu \mathrm{L}}{6}+\frac{\mathrm{k}_{2} \mathrm{~L}}{12} \mathrm{u}_{2}^{\mathrm{m}+1}+\frac{\mathrm{k}_{2} \mathrm{~L}}{12} \mathrm{u}_{3}^{\mathrm{m}+1}\right)^{(\mathrm{e})} \mathrm{v}_{3}^{\mathrm{m}} \\
& \mathrm{b}_{(\mathrm{n}-1) 1}=\left(\frac{(1-\theta) \mathrm{D}_{2}}{\mathrm{~L}}+\frac{\mathrm{L}}{6 \Delta \mathrm{t}}-\frac{\mu \mathrm{L}}{6}+\frac{\mathrm{k}_{2} \mathrm{~L}}{12} \mathrm{u}_{\mathrm{n}-2}^{\mathrm{m}+1}+\frac{\mathrm{k}_{2} \mathrm{~L}}{12} \mathrm{u}_{\mathrm{n}-1}^{\mathrm{m}+1}\right)^{(\mathrm{e})} \mathrm{v}_{\mathrm{n}-2}^{\mathrm{m}} \\
& +\left(\frac{-(1-\theta) D_{2}}{L}+\frac{2 L}{6 \Delta t}-\frac{\mu L}{3}+\frac{k_{2} L}{12} u_{n-2}^{m+1}+\frac{k_{2} L}{4} u_{n-1}^{m+1}\right)^{(e)} v_{n-1}^{m} \\
& +\left(\frac{-(1-\theta) D_{2}}{L}+\frac{2 L}{6 \Delta t}-\frac{\mu L}{3}+\frac{k_{2} L}{4} u_{n-1}^{m+1}+\frac{k_{2} L}{12} u_{n}^{m+1}\right)^{(e)} v_{n-1}^{m} \\
& +\left(\frac{(1-\theta) \mathrm{D}_{2}}{\mathrm{~L}}+\frac{\mathrm{L}}{6 \Delta \mathrm{t}}-\frac{\mu \mathrm{L}}{6}+\frac{\mathrm{k}_{2} \mathrm{~L}}{12} \mathrm{u}_{\mathrm{n}-1}^{\mathrm{m}+1}+\frac{\mathrm{k}_{2} \mathrm{~L}}{4} \mathrm{u}_{\mathrm{n}}^{\mathrm{m}+1}\right)^{(\mathrm{e})} \mathrm{v}_{\mathrm{n}}^{\mathrm{m}} \\
& b_{n 1}=\left(\frac{(1-\theta) D_{2}}{L}+\frac{L}{6 \Delta t}-\frac{\mu L}{6}+\frac{k_{2} L}{12} u_{n-1}^{m+1}+\frac{k_{2} L}{12} u_{n}^{m+1}\right)^{(e)} v_{n-1}^{m} \\
& +\left(\frac{-(1-\theta) \mathrm{D}_{2}}{\mathrm{~L}}+\frac{2 \mathrm{~L}}{6 \Delta \mathrm{t}}-\frac{\mu \mathrm{L}}{3}+\frac{\mathrm{k}_{2} \mathrm{~L}}{12} \mathrm{u}_{\mathrm{n}-1}^{\mathrm{m}+1}+\frac{\mathrm{k}_{2} \mathrm{~L}}{4} \mathrm{u}_{\mathrm{n}}^{\mathrm{m}+1}\right)^{(\mathrm{e})} \mathrm{v}_{\mathrm{n}}^{\mathrm{m}}
\end{aligned}
$$

we can solve the previous systems (37) and (38) by using the Gaussian elimination method.

\section{Numerical results:}

In this section, we have solved the systems (37) and (38) with the neumann boundary conditions [6]: 


$$
\begin{array}{ll}
\frac{\partial \mathrm{u}}{\partial \mathrm{x}}=0 & \text { at } \mathrm{x}=0 \text { and } \mathrm{x}=\mathrm{L} \\
\frac{\partial \mathrm{v}}{\partial \mathrm{x}}=0 & \text { at } \mathrm{x}=0 \text { and } \mathrm{x}=\mathrm{L}
\end{array}
$$

and initial condition [6]:

$\mathrm{u}(\mathrm{x}, 0)=\mathrm{f}(\mathrm{x})=\frac{1}{10} \sin ^{2}\left(\frac{24}{10} \pi \mathrm{x}\right)+\frac{28}{100} \sin ^{2}\left(\frac{-5}{100} \pi \mathrm{x}\right) \quad \mathrm{x} \in[0, \mathrm{~L}]$

$\mathrm{v}(\mathrm{x}, 0)=\mathrm{g}(\mathrm{x})=\frac{1}{10} \sin ^{2}\left(\frac{24}{10} \pi \mathrm{x}\right)+\frac{28}{100} \sin ^{2}\left(\frac{-5}{100} \pi \mathrm{x}\right) \quad \mathrm{x} \in[0, \mathrm{~L}]$

where step size $\mathrm{L}=0.05$ and time step $\Delta t=0.1, D_{1}=D_{2}=0.001, \alpha=1$, $k_{1}=k_{2}=1, \theta=0.5$ and $\mu=0.06$. The results are given in table (1) and fiqure (1).

When we take step size $\mathrm{L}=0.05$ and time step $\Delta t=0.1, D_{1}=D_{2}=0.001$, $\alpha=1, k_{1}=k_{2}=0, \theta=0.5$ and $\mu=0.01$. The results are given in table (2) and fiqure (2). 
Table (1). A comparison between the F.D.M. and Galerkin F.E.M. with the initial condition: $\mathrm{f}(\mathrm{x})=\mathrm{g}(\mathrm{x})=\frac{1}{10} \sin ^{2}\left(\frac{24}{10} \pi \mathrm{x}\right)+\frac{28}{100} \sin ^{2}\left(\frac{-5}{100} \pi \mathrm{x}\right)$.

\begin{tabular}{|c|c|c|c|}
\hline $\begin{array}{l}\text { Finite difference } \\
\text { method }\end{array}$ & $\begin{array}{l}\text { Galerkin finite } \\
\text { element method }\end{array}$ & $\begin{array}{l}\text { Finite difference } \\
\text { method }\end{array}$ & $\begin{array}{l}\text { Galerkin finite } \\
\text { element method }\end{array}$ \\
\hline$D_{1}=0.001, D_{2}=0.001$ & $D_{1}=0.001, D_{2}=0.001$ & $D_{1}=0.001, D_{2}=0.001$ & $D_{1}=0.001, D_{2}=0.00$ \\
\hline$k_{1}=1, k_{2}=1, \alpha=1$ & $k_{1}=1, k_{2}=1, \alpha=1$ & $k_{1}=1, k_{2}=1, \alpha=1$ & $k_{1}=1, k_{2}=1, \alpha=1$ \\
\hline$\mu=0.06, \theta=0$ & $\mu=0.06, \theta=0.5$ & $\mu=0.06, \theta=0$ & $\mu=0.06, \theta=0.5$ \\
\hline$L=0.05, \Delta t=0.1$ & $L=0.05, \Delta t=0.1$ & $L=0.05, \Delta t=0.1$ & $L=0.05, \Delta t=0.1$ \\
\hline $\mathrm{t}=14.5$ & $\mathrm{t}=14.5$ & $\mathrm{t}=8$ & $\mathrm{t}=8$ \\
\hline $\mathrm{u}(\mathrm{x}, \mathrm{t})$ & $\mathrm{u}(\mathrm{x}, \mathrm{t})$ & $\mathrm{v}(\mathrm{x}, \mathrm{t})$ & $\mathrm{v}(\mathrm{x}, \mathrm{t})$ \\
\hline 0.01727364 & 0.01695454 & 0.73037235 & 0.77554676 \\
\hline 0.01708765 & 0.01677521 & 0.78649215 & 0.81768448 \\
\hline 0.01662541 & 0.01632946 & 0.90756766 & 0.91319185 \\
\hline 0.01612475 & 0.01584698 & 1.01406856 & 1.00260765 \\
\hline 0.01583993 & 0.01557386 & 1.06219185 & 1.04482057 \\
\hline 0.01590271 & 0.01563745 & 1.04067279 & 1.02619394 \\
\hline 0.01624954 & 0.01597543 & 0.95552985 & 0.95602083 \\
\hline 0.01666076 & 0.01637400 & 0.83812229 & 0.86014518 \\
\hline 0.01688514 & 0.01658927 & 0.75844870 & 0.80048434 \\
\hline 0.01677429 & 0.01647741 & 0.78238088 & 0.81935633 \\
\hline 0.01635754 & 0.01606683 & 0.89132091 & 0.90468775 \\
\hline 0.01582614 & 0.01554364 & 1.00784963 & 1.00161710 \\
\hline 0.01543709 & 0.01515988 & 1.07730649 & 1.06259648 \\
\hline 0.01538274 & 0.01510713 & 1.08208726 & 1.06755464 \\
\hline 0.01569478 & 0.01542225 & 1.02331169 & 1.01715799 \\
\hline 0.01623924 & 0.01598064 & 0.91868160 & 0.93018756 \\
\hline 0.01680249 & 0.01657747 & 0.81536852 & 0.84587901 \\
\hline 0.01720984 & 0.01704017 & 0.77568034 & 0.80700317 \\
\hline 0.01740547 & 0.01730286 & 0.81304924 & 0.82317497 \\
\hline 0.01745113 & 0.01740520 & 0.87418656 & 0.88141190 \\
\hline 0.01745008 & 0.01742652 & 0.87151268 & 0.87991302 \\
\hline
\end{tabular}


Table (2). A comparison between the F.D.M. and Galerkin F.E.M. with the initial condition: $\mathrm{f}(\mathrm{x})=\mathrm{g}(\mathrm{x})=\frac{1}{10} \sin ^{2}\left(\frac{24}{10} \pi \mathrm{x}\right)+\frac{28}{100} \sin ^{2}\left(\frac{-5}{100} \pi \mathrm{x}\right)$.

\begin{tabular}{|c|c|c|c|}
\hline $\begin{array}{c}\begin{array}{c}\text { Finite difference } \\
\text { method }\end{array} \\
D_{1}=0.001, D_{2}=0.001 \\
k_{1}=0, k_{2}=0, \alpha=1 \\
\mu=0.01, \theta=0 \\
L=0.05, \Delta t=0.1 \\
\mathrm{t}=9.5 \\
\mathrm{u}(\mathrm{x}, \mathrm{t})\end{array}$ & $\begin{array}{c}\text { Galerkin finite } \\
\text { element method } \\
D_{1}=0.001, D_{2}=0.001 \\
k_{1}=0, k_{2}=0, \alpha=1 \\
\mu=0.01, \theta=0.5 \\
L=0.05, \Delta t=0.1 \\
\mathrm{t}=9.5 \\
\mathrm{u}(\mathrm{x}, \mathrm{t})\end{array}$ & $\begin{array}{c}\begin{array}{c}\text { Finite difference } \\
\text { method }\end{array} \\
D_{1}=0.001, D_{2}=0.001 \\
k_{1}=0, k_{2}=0, \alpha=1 \\
\mu=0.01, \theta=0 \\
L=0.05, \Delta t=0.1 \\
\mathrm{t}=13.5 \\
\mathrm{v}(\mathrm{x}, \mathrm{t})\end{array}$ & $\begin{array}{c}\text { Galerkin finite } \\
\text { element method } \\
D_{1}=0.001, D_{2}=0.001 \\
k_{1}=0, k_{2}=0, \alpha=1 \\
\mu=0.01, \theta=0.5 \\
L=0.05, \Delta t=0.1 \\
\mathrm{t}=13.5 \\
\mathrm{v}(\mathrm{x}, \mathrm{t})\end{array}$ \\
\hline 0.99940894 & 0.99945457 & 0.04189622 & 0.04110720 \\
\hline 0.99942310 & 0.99947123 & 0.04243666 & 0.04159035 \\
\hline 0.99945826 & 0.99951240 & 0.04377318 & 0.04368605 \\
\hline 0.99949645 & 0.99955657 & 0.04520580 & 0.04507065 \\
\hline 0.99951882 & 0.99958132 & 0.04599873 & 0.04578861 \\
\hline 0.99951566 & 0.99957533 & 0.04577925 & 0.04560786 \\
\hline 0.99949131 & 0.99954407 & 0.04473965 & 0.04469978 \\
\hline 0.99946153 & 0.99950661 & 0.04353281 & 0.04364617 \\
\hline 0.99944485 & 0.99948520 & 0.04291766 & 0.04312346 \\
\hline 0.99945266 & 0.99949328 & 0.04334606 & 0.04323447 \\
\hline 0.99948332 & 0.99952845 & 0.04471343 & 0.04468572 \\
\hline 0.99952344 & 0.99957422 & 0.04640577 & 0.04632790 \\
\hline 0.99955527 & 0.99960918 & 0.04761370 & 0.04743364 \\
\hline 0.9995658 & 0.99961824 & 0.04774012 & 0.04755889 \\
\hline 0.9995528 & 0.99959954 & 0.04668071 & 0.04659355 \\
\hline 0.99952469 & 0.99956406 & 0.04483322 & 0.04477505 \\
\hline 0.99949472 & 0.99952862 & 0.04284876 & 0.04277671 \\
\hline 0.99947379 & 0.99950634 & 0.04128434 & 0.04110823 \\
\hline 0.99946514 & 0.99950015 & 0.04036425 & 0.04020614 \\
\hline 0.99946458 & 0.99950323 & 0.03997844 & 0.03992289 \\
\hline 0.99946542 & 0.99950576 & 0.03988856 & 0.03979043 \\
\hline
\end{tabular}




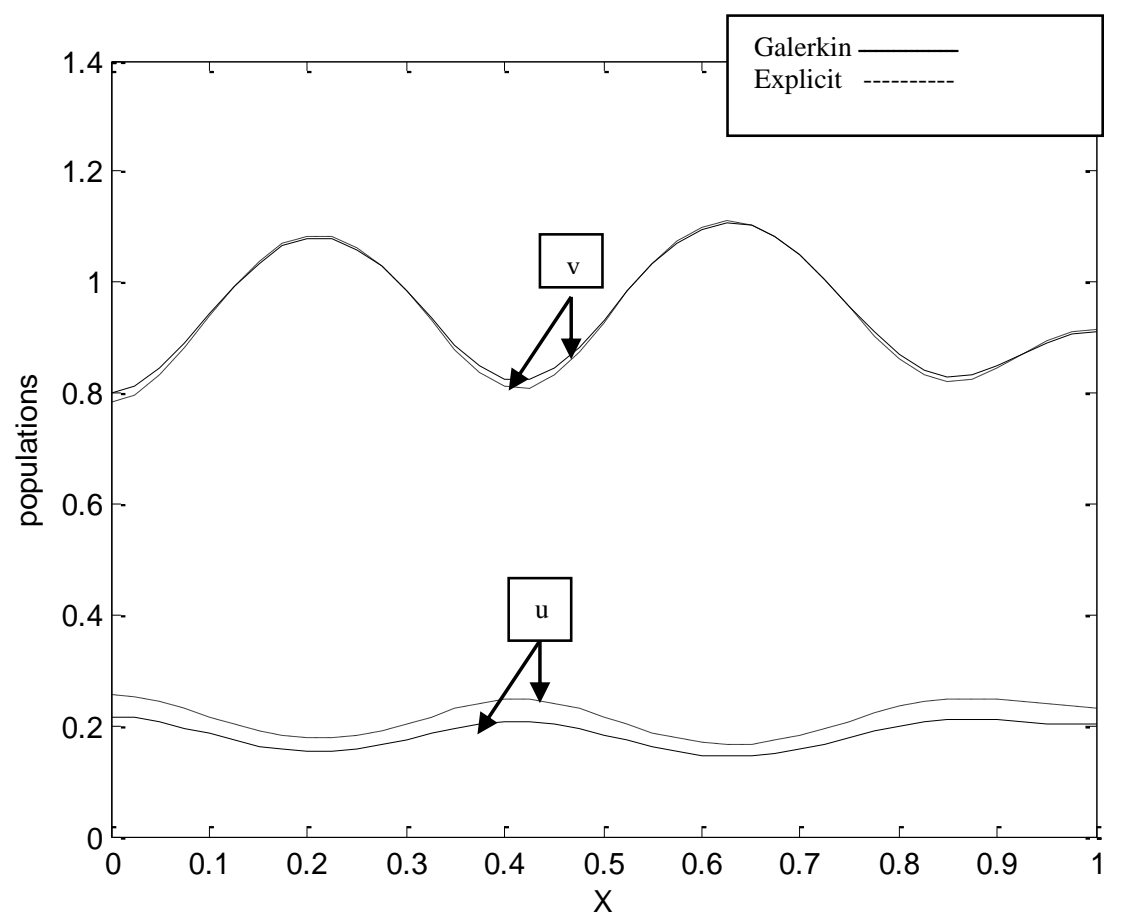

Figure (1). Comparison between the F.D.M. and Galerkin F.E.M. with the initial condition: $\mathrm{f}(\mathrm{x})=\mathrm{g}(\mathrm{x})=\frac{1}{10} \sin ^{2}\left(\frac{24}{10} \pi \mathrm{x}\right)+\frac{28}{100} \sin ^{2}\left(\frac{-5}{100} \pi \mathrm{x}\right)$.

Where $\alpha=1, D_{1}=D_{2}=0.001, k_{1}=k_{2}=1, \mu=0.06$ and $\mathbf{t}=\mathbf{1 0 . 5}$ 


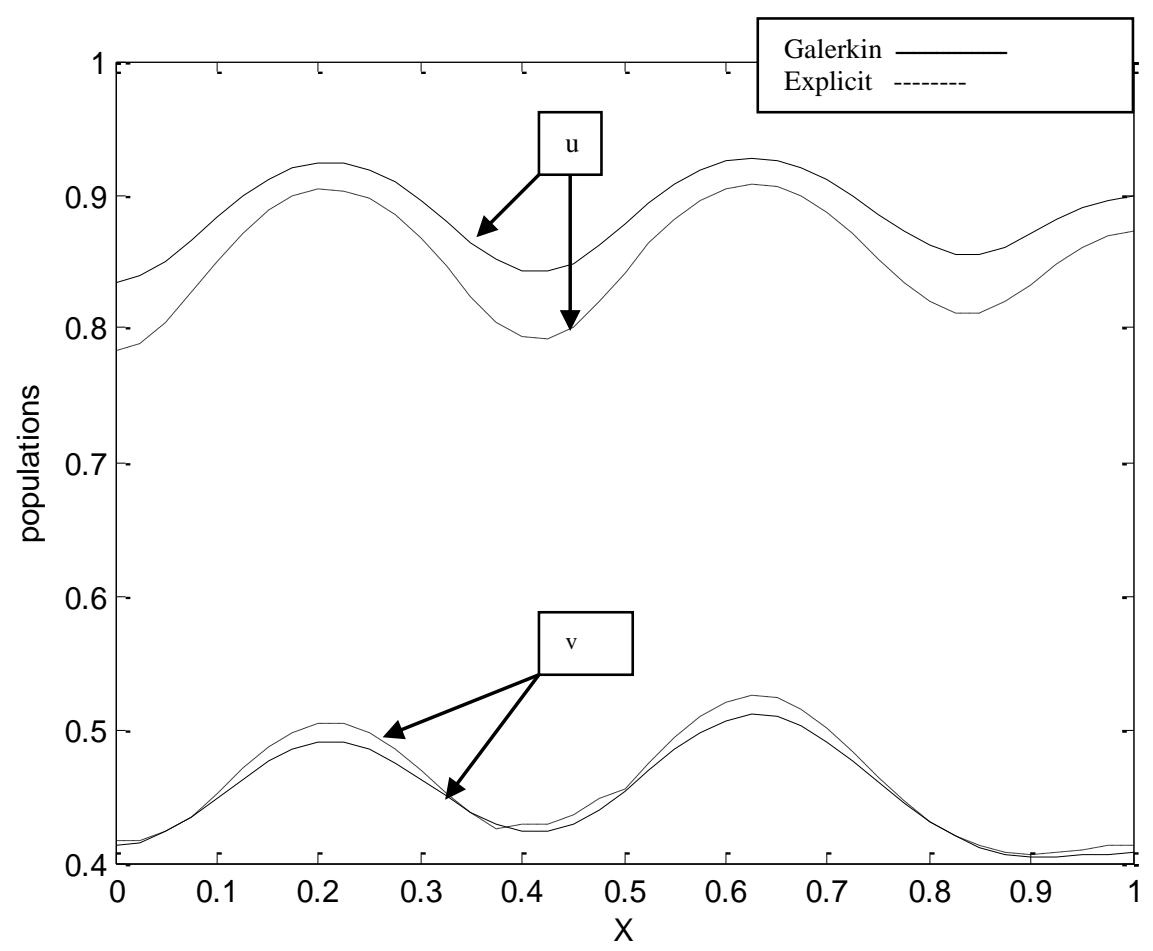

Figure (2). Comparison between the F.D.M. and Galerkin F.E.M. with the initial condition:

$\mathrm{f}(\mathrm{x})=\mathrm{g}(\mathrm{x})=\frac{1}{10} \sin ^{2}\left(\frac{24}{10} \pi \mathrm{x}\right)+\frac{28}{100} \sin ^{2}\left(\frac{-5}{100} \pi \mathrm{x}\right) \cdot$ Where
$\alpha=1, D_{1}=D_{2}=0.001, k_{1}=k_{2}=0, \mu=0.01$ and $\mathrm{t}=\mathbf{2 1 . 5}$

\section{Discussion:}

The main conclusion, which we can draw from this result is that Galerkin finite element method is faster than finite difference method to reach the equilibrium state and to reach the steady state solutions with less steps in time, from Fiqure (1) and table (1) it is obvious that Galerkin method is faster than finite difference method to reach the equilibrium state where the values for $\mathrm{u}(\mathrm{x}, \mathrm{t})$ are equal for all the values $(\mathrm{x})$ and $(\mathrm{t})$, and $\mathrm{v}(\mathrm{x}, \mathrm{t})$ is also equal for all the values ( $\mathrm{x}$ ) and ( $\mathrm{t}$ ). Also Galerkin method is faster than finite difference method to make the predator $v(x, t)$ reach the steady state solution $(\mathrm{v}=1)$, while the prey $\mathrm{u}(\mathrm{x}, \mathrm{t})$ decreases to steady state $(\mathrm{u}=0)$ in far time steps, given that the rate of interraction $\left(k_{1}=k_{2}=1\right)$ between the two species and the rate of death $(\mu=0.06)$ of the old predator, and the rate of growth $(\alpha=1)$ of the prey. 
With an interraction $\left(k_{1}=k_{2}=0\right)$ between the two species and the rate of death $(\mu=0.01)$ of the old predator, and the rate of growth $(\alpha=1)$ of the prey, Galerkin method faster than finite difference method to reach the prey $\mathrm{u}(\mathrm{x}, \mathrm{t})$ and the predator $\mathrm{v}(\mathrm{x}, \mathrm{t})$ to the equilibrium state and to make the prey reach a steady state $(\mathrm{u}=1)$ and the predator to steady state $(\mathrm{v}=0)$ in far time steps as shown in table (2) and figure (2). 


\section{REFERENCES}

[1] Allaire, P. E. (1985) Basics of the finite element method, Wn. C. Brown publishers.

[2] Arditi, R.; Tyutyunov, Y.; Morgulis, A. and Senina, I. (2001) "Directed movement of predators and the emergence of densitydependence in predator-prey models", Theoretical population biology 59, PP. 207-221.

[3] Desai, S. C. and Abel, J. F. (1972) Introduction to the finite element method, Van. Nostrand veinhold company.

[4] Du, Y. and Lou, Y. (1997) "Some uniqueness and exact multiplicity results for a predator-prey model", Transactions of the American Mathematical Society, Vol. 349, No. 6, PP. 2443-2475.

[5] Meng, Y. and Wang, Y. (2005) "Asymptotic behavior of a predatorprey diffusion system with time delays", Electronic journal of differential equations, Vol. 2005, No. 131, PP. 1-11.

[6] Nyaanga, P. P. (2004) "The interaction of species in population biology", www.ii.uib.no/ pius/thesis3.pdf.

[7] Segerlind, L. J. (1976) Applied finite element analysis, John wiley \& sons, Inc.

[8] Tortorell, V. D. Dimitrios, V. and Domiel, A. (2005) “A discontinuous Galerkin formulation for solution of parabolic equations on Nonconforming meshes",www.cims.nyu.edu/dd16/proceedings/ kulkarni_contrib.pdf. 